ten attackiert werden. Dies ist allerdings nur ein Vorgeplänkel für den unglückseligen 8. Juli 2016.

Am Morgen jenes Freitags, dem Vortag des fünften Unabhängigkeitsjubiläums, begibt sich Riek Machar zu einem von JMEC vermittelten Treffen mit Salva Kiir und Vizepräsident James Wani Igga in den Präsidentenpalast J1. In der Früh hatten noch beide Seiten zu einer sofortigen Einstellung erster Scharmützel aufgerufen. Ohne Erfolg. Kurz nach dem Eintreffen von Macher bricht vor dem Palast ein heftiges Feuergefecht zwischen den Wachkontingenten von Kiir und Machar aus. Das auf offener Straße ausgetragene Shoot-Out, dessen Spuren auch über vier Jahre später noch an den Wänden gegenüber von J1 sichtbar sind, kostet 150 Menschen das Leben. Riek Machar, zunächst von Salva Kiir höchstpersönlich gegen Kugeln abgeschirmt, gelingt es, zu seiner Basis hinter dem Jebel Kujur zu entkommen. Kurzfristig ist die Lage verworren. Dennoch deuten Augenzeugenberichte, die eine kurzfristige massive Verstärkung des den Präsidenten bewachenden Tiger DivisionKontingents vor dem Gefecht beobachtet haben wollen ${ }^{15}$, auf einen geplanten Charakter dieses Ereignisses hin.

\title{
Zusammenbruch und Revitalisierung
}

Die darauffolgenden Kämpfe in Juba dauern drei Tage und fordern mehrere hundert Todesopfer. Am 10. Juli kommt es zu einem Angriff auf Machars Hauptquartier, was dessen Entscheidung, in die Demokratische Republik Kongo (DRC) zu fliehen, mitauslösen dürfte. Der Trek in Richtung des Garamba Nationalparks in DRC dauert 30 Tage. Er wird von all jenen unternommen, die die Schlacht in Juba einigermaßen unbeschadet überstehen. Jene SPLA-IO-Kämpfer:innen, die verwundet zurückbleiben, suchen in den beiden von UNMISS bewachten PoC-Sites Schutz. Dies führt zu mitunter langen, persönlichen Trennungen. Im November 2020 treffe ich zufällig einen IO-Soldaten auf seinem ersten Besuch in Juba nach dem damaligen Treck. Plötzlich, gerade als er mir von seinen Erfahrungen auf dem Weg nach DRC berichtet, verliert er jede Contenance. Er springt auf und umarmt einen neu Hinzukommenden, minutenlang, unter Tränen. Es ist sein Bruder, der nach Verwundungen in Juba im PoC Camp in Juba zurückbleiben musste. Es war ihr erstes Wiedersehen nach über vier Jahren. 
Etwa tausend Getreue begleiten Machar auf seinem Treck, unter beständiger Bedrohung durch Regierungstruppen. Nach Ankunft im Garamba Nationalpark treten sie mit MONUSCO, der UN-Friedensmission in DRC, in Verhandlungen um Nahrungsmittelunterstützung und weitere humanitäre Hilfe. Unmittelbar danach beginnen Gespräche mit dem sudanesischen Regime, nach wie vor präsidiert von Omar al-Bashir, der in der letzten Phase seiner Herrschaft die IO als willkommene Verhandlungsmasse gegen die SPLM/A einsetzen will. Neben der eher passiven Duldung von darfurischen Rebellengruppen durch die SPLM/A geht es insbesondere um den Konflikt im "Neuen Süden« und die von der SPLM/A strategisch unterstützten Aktivitäten der SPLM-N in South Kordofan und Blue Nile, die Bashir untergraben will. Machar erreicht sein Ziel. Er und 150 seiner engsten Getreuen werden nach Khartum ausgeflogen. Was zu diesem Zeitpunkt aus Sicht der SPLM/A-IO alternativlos ist, wird sich mittelfristig jedoch aufgrund der immer deutlicher hervortretenden Eigeninteressen Khartums als problematisch herausstellen.

In der Tat kommt es bald nach dem endgültigen Zusammenbruch des Friedensprozesses zu einer Annäherung zwischen Sudan und Uganda. Yoweri Museveni war wegen der UPDF-Intervention in der ersten Phase des Bürgerkrieges massiver Kritik durch andere IGAD-Mitgliedsstaaten ausgesetzt. Nicht zuletzt deswegen entscheidet er sich diesmal gegen ein aktives Eingreifen und eine allzu offensichtliche Unterstützung von Salva Kiir. Dennoch ist Uganda nicht zuletzt wegen der massiven Fluchtbewegungen von Südsudanes:innen nach Norduganda an einer nachhaltigen Lösung interessiert. In den ersten Wochen nach dem neuerlichen Entflammen des Bürgerkrieges fliehen 300.000 Menschen über die Grenze. Dieser Konvergenz der Interessen der beiden Nachbarstaaten des Südsudan wird in den späteren Friedensverhandlungen eine entscheidende Rolle zukommen.

Die SPLM/A-IO geht geschwächt in die neue Phase des Bürgerkrieges. Zwar war es ihr gelungen, während des Marsches nach DRC eine gewisse Unterstützung in Western Equatoria zu generieren. Zugleich verlor sie aber im Zuge dieser neuerlichen Zuspitzung ihre letzten Verbindungen zu den DinkaGemeinschaften. Diese Entwicklung hat auch in Hinblick auf die Institutionalisierung des südsudanesischen politischen Systems problematische Auswirkungen. Trotz der aktiven Versuche beider Seiten, multiethnische Koalitionen zu schmieden, nimmt die ethnopolitische Polarisierung zwischen einer als Dinka-lastig wahrgenommenen Regierung und einer von Nuer und einigen Shilluk dominierten Opposition weiter zu. Zugleich koppelt sich das in dieser Polarisierung nicht repräsentierte Equatoria von der Auseinander- 
setzung weiter ab. Militärisch ist die Position der IO ebenfalls geschwächt, insbesondere in Upper Nile kommt es wiederholt zu Rückschlägen. Als effektivste Kraft erweist sich Johnson Olonyis Agwelek Division, die die ShillukGebiete westlich des Nil wieder unter IO-Kontrolle bringen kann.

Ebenso kann Machar eine Spaltung der IO, die schon länger befürchtet worden war, nicht verhindern. Taban Deng stellt sich offen gegen die MacharFraktion und übernimmt wenige Wochen nach dem »Juba Incident« am 26. Juli 2016 auf Salva Kiirs Angebot hin Machars Position als Erster Vizepräsident. Taban Dengs Worte - »This country has a constitution, this country has a president and has a law to be followed. Your Excellence Mr. President, as I said, you are my commander in chief « - sind ebenso eindeutig wie jene Salva Kiirs: „Of course this agreement cannot be personalized that if $\mathrm{X}$ is away, the agreement can be shelved until when that person comes «. ${ }^{16}$ Diese Position wird von der Obama-Administration in ihrem verbissenen Versuch, den Friedensprozess wider alle gegenteiligen Anzeichen $\mathrm{zu}$ retten, gegen die ursprüngliche Intention des ARCSS offiziell unterstützt. Während eines Staatsbesuches in Kenia im August 2016 gibt Secretary of State John Kerry zu Protokoll: »I think it's quite clear that legally, under the agreement, there is allowance for the replacement in a transition of personnel, and that has been effected with the appointment of a new vice president. ${ }^{17}$ Letzten Endes bleibt auch der um vieles skeptischer eingestellten IGAD nichts anderes übrig, als die geschaffenen Fakten zu akzeptieren.

Auf Regierungsseite kommt es ebenfalls zu Komplikationen. Offenbar als Antwort auf massives Lobbying, mutmaßlich aus radikalen Dinka-Kreisen, erweitert Kiir im Januar 2017 die Zahl der Gliedstaaten neuerlich, von 28 auf 32, was zu formalen Protesten von UN und IGAD führt. Am 10. Februar 2017 tritt Thomas Cirillo Swaka, einer der Stellvertretenden Oberkommandierenden der SPLA, von seiner Position zurück. Er bezichtigt Generalstabschef Paul Malong des Tribalismus und der bewussten Torpedierung des Friedensprozesses (»orchestrated by design « lautet die von ihm gewählte Formulierung ${ }^{18}$ ). Cirillo, als fähiger und konsequenter Kommandant bekannt, begibt sich umgehend in die Gegend von Yei im südlichen Central Equatoria und formiert eine Miliz unter dem Namen National Salvation Front (NAS). Die NAS erweist

\footnotetext{
16 https://sudantribune.com/spip.php?article59735 [17-01-2021].

17 https://2009-2017.state.gov/secretary/remarks/2016/08/261188.htm [17-01-2021].

18 https://www.malakalpost.com/lt-gen-thomasEdwardThomas-cirillo-swakaThomasCiril loSwaka-the-deputy-chief-of-general-staff-for-logistics-resignation-letter [17-01-2021].
} 
sich in ihren Auseinandersetzungen mit der SPLM/A und der späteren SSPDF als militärisch effektiv, politisch stringent und wenig kompromissbereit. Bald erweitert sie ihre Aktivitäten auf das gesamte Equatoria. Auch eine relevante Zahl an SPLM/A-IO-Kadern in der Region schließt sich der NAS an.

Die sich verschärfende Krise in den Equatorias beantwortet Salva Kiir mit überraschend deutlichen und politisch riskanten Einschnitten. Am 9. Mai entlässt er Generalstabschef Paul Malong und ersetzt ihn durch James Ajongo Mawut. Offenbar hat Malong, der unter Einsatz der von ihm orchestrierten Dinka-Miliz Mathiang Anyoor wesentlich zum Ausbruch des Bürgerkrieges beigetragen hat und als der prominenteste Gegner jedes Kompromisses mit der SPLM/A-IO gilt, seine Karten überspielt. Vermutlich nicht zu Unrecht werden ihm politische Ambitionen auf die Position Kiirs nachgesagt. Malong macht sich umgehend mit einer starken Leibwache auf den Weg in seine Heimatgemeinde Aweil. Angesichts einer präsidentiellen Order, seine Ankunft zu unterbinden, lässt sich Malong nach längeren Gesprächen in Yirol, in denen ihm unter anderem freies Geleit zugesichert wird, zu einer Rückkehr nach Juba umstimmen. In weiterer Folge begibt er sich ins Exil nach Kenia. In Nairobi beginnt er umgehend mit dem Aufbau einer eigenen bewaffneten Formation, der South Sudan United Front (SSUF). Die SSUF kann auf der lokalen Popularität des von den UN wegen mutmaßlicher Kriegsverbrechen sanktionierten und steinreichen Malong aufbauen.

Die militärische Führung findet nach Malongs Abgang in keine ruhigen Fahrwasser. Zunächst wird die SPLA in South Sudanese Defence Forces (SSDF), und schließlich, im August 2017, in South Sudanese People's Defence Forces (SSPDF) umbenannt. Bei den Oberkommandierenden kommt es zu raschen Wechseln. Malongs Nachfolger, James Ajongo, erkrankt und verstirbt weniger als ein Jahr nach Amtsübernahme. Ihm folgt der wie Malong ebenfalls von den UN sanktionierte Bor Dinka Gabriel John Riak. Dieser wird nach weiteren zwei Jahren, im Mai 2020, wiederum von Johnson Juma Okot abgelöst. Johnson Juma, ein Acholi aus Eastern Equatoria, wird vermutlich nicht zuletzt wegen der Notwendigkeit einer stärkeren Repräsentanz Equatorias in der SSPDF-Führung für diese Funktion ausgewählt.

Zugleich verändert die Entlassung Malongs die politische Dynamik in Juba. In die Rolle des neuen zentralen Partners - und Kontrahenten - von Kiir schlüpft der Direktor des Internal Security Bureau im National Security Service (NSS), Akol Koor Kuc. Wie Kiir stammt Akol Koor aus Warrap, und er verdankt Kiir zu wesentlichen Stücken seine Karriere. Unmittelbar nach der Unabhängigkeit befördert Kiir den in niederen Rängen stehenden Offizier di- 
rekt zum Generalleutnant und macht ihn als Leiter des Internal Security Bureau zum faktischen Chef aller südsudanesischen Geheimdienste. Akol Koor wird es ihm in den folgenden Jahren durch bedingungslose Loyalität und die konsequente Verfolgung aller oppositionellen Strömungen vergelten. Wenig überraschend wird Koor eine wesentliche Rolle bei der Entlassung Malongs zugeschrieben.

Zusätzlich zu seiner Funktion im NSS fungiert Koor lange als Vorstandsmitglied des sudanesischen Ölkonsortiums Nilepet, wobei er einen Teil des dadurch kontrollierten Geldes nachweislich für Waffeneinkäufe zugunsten außerhalb der SPLA operierender Dinka-Milizen verwendet. Die immer einflussreichere Rolle von Koor, und des NSS im Allgemeinen, führt zu zunehmenden Spannungen mit der SPLA und der späteren SSPDF. Auch das Verhältnis zu Kiir wird offenbar fragiler. Im September 2020 entfernt Kiir Koor und den Generaldirektor des General Intelligence Bureau im NSS, Thomas Duoth Guet, von ihren Positionen bei Nilepet, offenbar in einem Versuch, die Macht des NSS im internen Gefüge zurückzubinden.

Trotz dieser politischen Herausforderungen und der aus den Fugen geratenden Situation in Equatoria dominiert die Regierungsseite den militärischen Konflikt mit der SPLM/A-IO. Die gegen das IO-Hauptquartier gerichtete Pagak Offensive im Juli und August 2017 versetzt der IO einen schweren militärischen und politischen Schlag, nicht zuletzt, da sich daran viele zu Taban Dengs IO-Regierungsfraktion übergelaufene, vormals eigene Kämpfer:innen beteiligen. Nach heftigen Kämpfen kann die SSPDF Pagak einnehmen. Damit untermauert sie ihre strategische Kontrolle des Landes, zu diesem Zeitpunkt sind bereits alle 32 Provinzhauptstädte unter Regierungskontrolle.

Zugleich steigt für beide Seiten der Druck, sich auf einen erneuten Verhandlungsprozess einzulassen. Das Personal der Trump-Administration tritt der SPLM um einiges kritischer gegenüber, als diese es aus Obama-Zeiten gewohnt war. Nikki Haley, US-amerikanische Botschafterin bei den UN, besucht Juba im Oktober 2017. Sie muss prompt wegen einer Anti-Kiir-Demonstration aus einem der PoC-Camps bei Juba evakuiert werden. Wenig später kritisiert sie die südsudanesische Regierung für deren Gewaltanwendung gegen die Zivilbevölkerung und agiert als treibende Kraft für die Verhängung eines internationalen Waffenembargos durch den UN-Sicherheitsrat mit UNSCR 2428 vom 13. Juli 2018.

Doch der am Ende entscheidende Druck zur Aufnahme von Friedensverhandlungen kommt nicht vom Westen, sondern von den beiden Nachbarn im Norden und im Süden, Sudan und Uganda, die sich mittlerweile auf eine 
gemeinsame strategische Vorgangsweise verständigt haben. Omar al-Bashir drängt Riek Machar bald nach seiner Ankunft in Khartum zur Teilnahme an ersten Vorverhandlungen in Addis Abeba. Nachdem der vorangegangene Friedensprozess formell nie beendet worden war, geht es nun um seine Revitalisierung. Von 18. bis 22. Dezember treffen sich die Parteien - neben dem TGoNU, das neben der SPLM/A auch die IO-Fraktion von Taban Deng umfasst, und der SPLM/A-IO noch die SPLM-FD, die NAS, das von Lam Akol geführte NDM, Peter Gadets SSUM, sowie weitere kleinere Milizen, die sich im Fortlauf der Verhandlungen zur South Sudan Opposition Alliance (SSOA) zusammenschließen werden - zu Waffenstillstandsverhandlungen in Addis Abeba. Ein erstes Waffenstillstandsabkommen wird unterzeichnet ${ }^{19}$, allerdings von Regierungsseite am Weihnachtstag mit Angriffen auf IO-Stellungen in Upper Nile und in Central Equatoria prompt wieder gebrochen.

Der Sudan ist gewillt, die Verhandlungen mit allen Mitteln voranzutreiben. Im Rahmen einer informellen Übereinkunft mit Südafrika befindet sich Riek Machar während der gesamten Verhandlungsmonate in faktischem Hausarrest in Johannesburg. Er kann nur zu den Gesprächen nach Khartum oder Addis Abeba ausreisen. Die wesentliche Motivation für den in den letzten Monaten seiner Herrschaft stehenden Omar al-Bashir in Khartum ist die Aussicht auf eine Wiederaufnahme der Ölförderung, die dringend benötigte Devisen, etwa über die Transitgebühren, in die sudanesischen Taschen spülen würden. Ein Friedensschluss wäre die Voraussetzung, diese Gelder zu lukrieren, und Bashir scheint zu spüren, dass in diesen Verhandlungen auch für ihn persönlich sehr viel auf dem Spiel steht. Uganda, das mittlerweile eine Million aus dem Südsudan Geflüchtete beherbergt, hat ebenso materielle Interessen an einem Friedensschluss und erhöht sukzessive den Druck auf Salva Kiir.

Zwar hat IGAD die offizielle Verhandlungsführung, doch die eigentliche Initiative kommt eindeutig aus Khartum. Die internationalen Mechanismen der ARCSS-Verhandlungen, IGAD PLUS und die Troika, sind zu diesem Zeitpunkt nicht mehr aktiv involviert. Im Juni 2018 findet eine erste substanzielle Verhandlungsrunde in Addis Abeba unter Beteiligung von Bashir und Museveni statt. Im Anschluss lädt Bashir die Parteien nach Khartum ein. Dort unterschreiben Kiir, Machar, die SSOA, die SPLM-FD und die Gruppe der OPP,

19 Agreement on Cessation of Hostilities, Protection of Civilians and Humanitarian Access, Republic of South Sudan, unterzeichnet am 21. Dezember 2017 in Addis Abeba, https://www.peaceagreements.org/viewmasterdocument/1966. 
die mittlerweile wieder zum Prozess gestoßen ist, unter Augen der IGAD eine Khartoum Declaration ${ }^{20}$, die den Waffenstillstand erneuert und in ihrem Hauptteil die Wiederaufnahme der Ölförderung in Unity State behandelt. Omar al-Bashir tritt als offizieller Garant des Vertrages auf.

Wenig später, am 7. Juli 2018, kommt es zum entscheidenden Tag dieser im Vergleich zum vorangegangenen Prozess grundlegend anders gestalteten Verhandlungen. Bashir besucht zusammen mit Kiir und Machar Yoweri Museveni in Entebbe nahe Kampala. In weit in die Nacht dauernden Verhandlungen werden die Grundzüge jenes Deals ausgehandelt, der wenig später in Addis Abeba unter Ägide der IGAD und unter Beteiligung der anderen Parteien formalisiert wird. Am 12. September 2018 wird das revitalisierte Friedensabkommen, R-ARCSS, schließlich von Salva Kiir, Riek Machar, Deng Alor (für SPLM-FD) und Gabriel Changson Chang (für SSOA), sowie sechs Vertretern der OPP unterzeichnet.

Die wohl wesentlichste politische Umwälzung in dieser finalen Phase der Verhandlungen ist das Auseinanderbrechen der SSOA. Thomas Cirillo verlässt mit einer Mehrheitsfraktion der NAS im Verein mit Lam Akols NDM und einzelnen anderen Gruppierungen das Oppositionsbündnis und bricht damit mit dem R-ARCSS-Prozess. Die Gruppierungen formieren sich neu als South Sudan Opposition Movement Alliance (SSOMA). Die SSOMA nimmt in der zweiten Jahreshälfte einen eigenen, von der Comunità di Sant'Egidio vermittelten Friedensprozess mit der Regierung auf, den sogenannten Rom-Prozess. Dieser Prozess hat, abgesehen von einer allgemeinen Deklaration ${ }^{21}$ und nicht eingehaltenen Waffenstillstandsabkommen, bislang zu keinen nachhaltigen Resultaten geführt. Jedoch kommt es im Zuge dieses Prozesses zu einer paradox erscheinenden Annäherung der zwei früheren Erzfeinde Cirillo und Malong. Malongs neu formierte SSUF wird in die SSOMA aufgenommen. Diese Eingliederung bleibt fragil und ist von Streitigkeiten überschattet.

Nach dem Friedensschluss setzt zunächst ein kollektives Durchatmen ein. Ende Oktober 2018 macht sich Machar auf den Weg nach Juba, verweilt dort aber in Anbetracht der nach wie vor volatilen Situation nur kurz. Wenig später

20 Khartoum Declaration of Agreement Between Parties of the Conflict of South Sudan, unterzeichnet am 27. Juni 2018 in Khartum, https://www.peaceagreements.org/viewm asterdocument/2109. 
reist er zurück nach Khartum. Dort findet er nach dem Sturz von Omar elBashir im April 2019 eine grundlegend veränderte politische Situation vor, die den auf ihm persönlich lastenden politischen Druck von sudanesischer Seite signifikant verringert. Auch die SPLM-FD findet sich wieder in Juba ein, um sich am Transitionsprozess zu beteiligen. Diese politische Transition bildet für sich genommen eine komplexe Konfliktlandschaft, die im Detail in einem späteren Kapitel behandelt wird.

Durch die ausschließliche Dominanz regionaler Akteure, insbesondere Sudans und Ugandas, die die breite internationale Beteiligung am ARCSSProzess konterkariert, nimmt der R-ARCSS in den afrikanischen Friedensprozessen eine Sonderstellung ein. Die noch aus CPA-Zeiten herrührende und bis zu diesem Zeitpunkt immer eingebundene Troika, bestehend aus den USA, Großbritannien und Norwegen, ist in den Verhandlungsprozess nicht eingebunden und über dessen Fortgang weitgehend uninformiert (ICG, 2019: 10). Ich selbst erlebte aufgrund eines glücklichen Zufalls die Meldung des Verhandlungsdurchbruchs in der Britischen High Commission in Nairobi, gemeinsam mit dem britischen Südsudan-Team, das sich von der letztendlichen Vertragsunterzeichnung genauso überrascht zeigt wie unbedarfte externe Beobachter:innen. In offiziellen Stellungnahmen zeigt sich die Troika von R-ARCSS zunächst wenig überzeugt. Erst spät bekennt sie sich zum Prozess und akzeptiert eine aktive Involvierung in dessen Implementierung.

Die Perspektiven des Abkommens sind umstritten und der mit dem Abkommen eingeleitete Transformationsprozess, wie wir noch sehen werden, herausfordernd. Dennoch zeichnet sich R-ARCSS gegenüber dem vorangegangenen ARCSS durch zwei wesentliche Unterschiede aus. Erstens ist der Vertrag ein Resultat eines genuinen regionalen Interesses, das von den zwei über die Jahrzehnte wesentlichsten Partnern der südsudanesischen Konfliktparteien durchgesetzt wird. Zweitens hat sich die militärische Lage noch weiter zugunsten der Regierung verschoben, was für die Vertragsumsetzung mindestens ebenso bedeutsam ist. Damit sinkt zwar die Kompromissbereitschaft der Regierung um Kiir, es sind aber intern weniger herausfordernde Stimmen zu erwarten. Der SPLM/A-IO wiederum bleibt, wie den anderen Unterzeichnern des Abkommens, keine andere Option, als den Prozess mit all seinen Schwierigkeiten durchzustehen und die bestmöglichen Resultate zu erzielen.

Am Ende dieser Abhandlung stellt sich naturgemäß die Frage, welche ersten Schlüsse aus dieser überaus turbulenten (de Waal et al., 2019: 2) politisch-militärischen Geschichte gezogen werden können, an deren Ende der 
Südsudan als unabhängiger, nun selbst in einen Friedensprozess verwickelter Staat steht. Es sind zwei Aspekte, die besondere Beachtung verdienen. Erstens zeigt sich die Wirkmächtigkeit kolonial institutionalisierter Diskurse und Gegensätze. Der tragische Gegensatz zwischen einer Polarisierung entlang des karikaturesken »Afrika" versus »Arabien«-Schemas einerseits und einer sich immer öfter gewaltsam entladenden »ethnischen« Auseinandersetzung im Inneren andererseits ist ohne die Kolonialgeschichte nicht erklärlich. Die Institutionalisierung und die damit einhergehende faktische Wirkungsmacht dieser Diskurse ist allerdings ebenso ein Produkt der politisch-militärischen Konflikte seit der sudanesischen Unabhängigkeit. Dies ist immer vor dem Hintergrund zu sehen, dass ein Prozess der nationalstaatlichen Vergesellschaftung im Gebiet des heutigen Südsudan nie stattgefunden hat.

Als eine zweite Erkenntnis ist festzuhalten, dass die allzu gerne gesuchten klaren politischen Zuschreibungen - "New Sudan« versus »Unabhängigkeit«, demokratisch versus diktatorisch, für oder gegen einen Friedens- oder Versöhnungsprozess - bei nahezu allen Exponent:innen südsudanesischer Politik versagen. Sie entspringen mehr den Ideen engagierter Kommentator:innen und deren, verständlichem, Wunsch nach Orientierung und Klarheit. Ironischerweise finden manche dieser äußeren Zuschreibungen tatsächlich Eingang in den südsudanesischen Diskurs - so ist die »New Sudan«-Vision niemals zuvor so eindeutig formuliert wie im SPLM Manifesto von 2008, also zu einem Zeitpunkt, an dem die nationale Unabhängigkeit längst zur einzigen gangbaren Option geworden war. Was bei aller militärischen Brillanz und Leistungsfähigkeit der SPLM/A, die in der lange Jahre für vollkommen unmöglich gehaltenen Unabhängigkeit mündet, vorherrscht, ist alles andere als die von außen gesuchte Klarheit. Es ist vielmehr taktisches Abwägen, Abwarten und strategische Ambivalenz. Es wäre jedoch ein grober Fehler, diese politischen Charakteristika als Schwäche misszuverstehen. 
